\title{
LOS CONFLICTOS SOCIO AMBIENTALES EN EL CORREDOR MINERO SUR ANDINO Y LOS MECANISMOS ALTERNATIVOS DE SOLUCIÓN
}

\author{
CORREDOR MINERO SUR ANDINO NISQAMANTA MAMAPACHAPI \\ CH'AQRUY SASAMANTA LLIW IMAYMANA NIRAQ \\ ALLICHAYMANTA
}

\section{THE SOCIO-ENVIRONMENTAL CONFLICTS IN THE SOUTHEASTERN MINING CORRIDOR AND THE ALTERNATIVE SOLUTION MECHANISMS}

\section{Antonio Eliseo Huañahui Sillocca(1)}

Ch'uyanchakuy: Peru suyupi aska conflictos socioambientales kan, chay conflicto sociales nisqamanta, chay kuna astawan kan ayllu minawan harkakuypi. Aska apurimac, Qosqo ayllunkuna ñawpa watakunamanta mineriawan kawsanku, uywakunawan, comercionisqawan. Cotabambas ayllu (Apurimaq), Espinar Chubivilcas ayllu (Qosqo), allpanku tupanku, chay raykun chapi kan corredor minero del sur andino nisqanku, chaypi rikukun Peru suyupa economian kallpachaqa. Kay articuloqa chay conflictos minerosmanta riman, Chumbivilcas ayllumanta, yachapakuy ruwaykunawan allichanapaq.

Yuyaysapa simikuna:T'irakuy, Allinchay, Pacha Mama, Kamachi.

Resumen: La evolución de los conflictos sociales en el Perú se ha caracterizado en los últimos años por un alto componente de conflictos socio ambientales que están relacionados mayormente a desencuentros entre las comunidades campesinas y la actividad minera, así como la imposición de un modo de hacer la minería y la forma inadecuada de relacionarse con su entorno. Numerosas poblaciones y comunidades campesinas de las regiones de Apurímac y Cusco tienen una larga trayectoria de coexistencia entre la minería, las actividades Ganaderas - Agrícolas y el Comercio. Las provincias Alto Andinas de Cotabambas

(1) Abogado por la Universidad Nacional de San Antonio Abad del Cusco, con estudios de Post Grado: Maestría en Derecho Constitucional y Doctorado en Derecho (Egresado), Profesor Universitario en la Especialidad de Constitución y Derechos Humanos de la UNSAAC. husianel@hotmail.com 
(Apurímac) y las provincias de Chumbivilcas y Espinar (Cusco), por ser colindantes y estar integradas geográfica, social y económicamente constituyen un corredor minero del sur andino, como una nueva zona de expansión minera en el país, la cual concentrará la mayor parte del impulso económico. Este artículo trata, sobre los conflictos mineros en la Provincia de Chumbivilcas y propone mecanismos alternativos de solución.

Palabras clave: Conflictos mineros, Conflictos socio ambientales, Minería, Forma de relacionamiento, Mecanismos alternativos de solución.

Abstract: The evolution of social conflicts in Peru has been characterized in recent years by a high component of socio-environmental conflicts mainly related to disagreements between peasant communities and mining activity, as well as the imposition of a way of doing mining and the inadequate way of relating to their environment. Numerous towns and rural communities in the regions of Apurimac and Cusco have a long history of coexistence between mining, Livestock - Agricultural activities and Trade. The provinces of Cotabambas (Apurímac) and the provinces of Chumbivilcas and Espinar (Cusco), because they are adjacent and geographically, socially and economically integrated, constitute a mining corridor in the Andean south, as a new zone of mining expansion in the country, which concentrate most of the economic momentum. This article deals with the mining conflicts in the Province of Chumbivilcas and proposes alternative mechanisms of solution.

Key words: Mining conflicts, Socio-environmental conflicts, How to do mining, Form of relationship, Alternative mechanisms of solution. 


\section{Introducción}

Las provincias de Cotabambas (Apurímac) y las provincias de Chumbivilcas y Espinar (Cusco), por ser colindantes y estar integradas geográfica, social y económicamente constituyen un corredor minero del sur andino, como una nueva zona de expansión minera en el país, la cual concentrará la mayor parte del impulso económico.

Numerosas poblaciones y comunidades campesinas de las regiones de Apurímac y Cusco llevan una larga trayectoria de coexistencia entre la minería, las actividades Ganaderas - Agrícolas y el Comercio.

La Provincia de Chumbivilcas, en la década del noventa era considerada como despensa minera para el Perú, lo que ha motivado el interés en las concesiones de sus territorios a favor de explotaciones mineras, con un alto porcentaje a nivel regional y nacional. A la fecha existen como proyectos mineros; Constancia, Azuca, Crespo, Anabi, Victoria, Winicocha, Katanga Este y otros en explotación y exploración. También se ve una fuerte presencia de la actividad minera informal e ilegal.

Según estimaciones del Ministerio de Energía y Minas, la inversión proyectada en nuevas explotaciones de minerales bordea la suma de 60 mil millones de dólares americanos para el período o década del 2016 - 2026 y la mitad de estas inversiones se encuentra en el Sur Andino Peruano, entre ellos Las Bambas en la Región Apurímac, Constancia, Las Quechuas, Coroccohuaycco, Antapaccay y otros en la Región Cusco.

Toda inversión minera, para poder cumplir sus objetivos de extracción, requiere de la pacífica relación con las poblaciones y comunidades campesinas, para lo cual toda negociación debe tomar en cuenta: los objetivos de los dueños de la tierra, de los inversionistas que con su tecnología e inmensos recursos monetarios van a explotar; y también los del Estado que con su recaudación tributaria puede desarrollar su objetivo fundamental: el bienestar de todos los peruanos.

\section{Condiciones fundamentales para la actividad minera}

Para que la minería y las industrias extractivas puedan realizarse deben cumplirse algunas condiciones fundamentales, que detallaremos en relación a las minas ya existentes en la relación con las nuevas operaciones que se quieren realizar, en torno a cuatro aspectos fundamentales:

1. Los planes de desarrollo territoriales y la zonificación económica-ecológica.

2. Los derechos de propiedades y uso de la tierra.

3. Las condiciones de preservación del medio ambiente y los recursos naturales.

4. La participación y consentimiento de las comunidades afectadas. 


\subsection{Minas existentes}

Las regulaciones en relación a las Minas ya existentes deben reconocer que éstas ya están en operación y buscar limitar sus efectos negativos. Las ampliaciones de las explotaciones mineras que representen grandes cambios deberán someterse a las regulaciones para nuevas mineras. En este caso, ya existiendo la minería, es necesario:

1. La elaboración y aprobación participativa de planes de desarrollo regionales, locales y comunales que incluyen estrategias y acciones para orientar la minería que ya está en operación y sus probables ampliaciones.

2. Una revisión para restablecer condiciones de justicia para los legítimos propietarios de la tierra.

3. Renegociar los acuerdos existentes entre las empresas mineras y las comunidades.

4. Revisar las relaciones entre Estado, empresa y comunidades para asegurar que la minería aporte al desarrollo comunal, local y regional.

5. La necesidad de que exista una autoridad de fiscalización autónoma.

\subsection{Nuevas Minas}

En relación con establecimiento de nuevas explotaciones mineras es necesario ser mucho más cuidadoso respecto de las condiciones bajo las cuales puedan desarrollarse, ésto para evitar casos en los que la explotación minera genere daños ambientales y sociales, severos y violente derechos fundamentales, retrasando el desarrollo local y regional. Entonces tenemos como cuestiones fundamentales a plantearse:

1. Sobre la base de los planes de desarrollo concertados y participativos, dentro de una lógica descentralista y ambiental, con criterios de cuenca y de resguardo de la biodiversidad que planifique y apruebe un ordenamiento territorial, se debe establecer una Zonificación Económica - Ecológica (ZEE) que establezca en qué territorios puede desarrollarse la minería y cuáles deben estar reservados y otros usos, sean éstas de preservación de la naturaleza, agropecuarias, turísticos u otros.

2. Revisar la forma de otorgamiento de las concesiones que otorgan derechos legales exclusivos de exploración y explotación a quiénes las obtienen, se deben conferir solamente en aquellos territorios calificados de acuerdo a la Zonificación Económica - Ecológica - ZEE como aptos para la explotación minera. Una revisión de los procedimientos y otros requisitos para las concesiones también es necesario ya que han sido otorgadas, en caso de Chumbivilcas, hasta el 2009, un total de 713 Concesiones (93\% de su territorio).

3. Los permisos de exploración y explotación deben otorgarse solamente donde la Zonificación Económica-Ecológica - ZEE, lo permita y requerir un acuerdo previo con los propietarios de la tierra, por lo cual debe derogarse el D. Leg. No 1064 que no contempla esa posibilidad. 
4. La participación y consulta con las comunidades de la zona deben ser reforzadas, respetándose el derecho al consentimiento previo, libre e informado establecido en el Convenio 169 de la OIT y las Declaración de las Naciones Unidas sobre los Pueblos Indígenas.

5. Fortalecimiento de capacidades de las comunidades, organizaciones de la sociedad civil y organizaciones de las comunidades indígenas y las comunidades afectadas por la minería.

6. La realización de consultas ciudadanas bajo diversos mecanismos por parte de comunidades y municipalidades debe ser respetada e incluso alentada como un mecanismo de consulta legítima, debiendo recibir apoyo de organismos estatales como la ONPE ${ }^{(2)}$.

7. La sostenibilidad ambiental debe ser asegurada. La situación actual no garantiza ello ya que las mismas empresas hacen los estudios.

8. Es necesario que los estudios de impacto ambiental (EIA) sean hechos por instituciones ajenas a las Empresas Mineras, bajo control directo del Estado.

9. Los estudios de impacto ambiental (EIAs) deben tener una línea de base independiente y un balance hídrico adecuado.

10. El Estado deberá también realizar estudios de impacto ambiental (EIA) estratégicos, cuando la cesión de explotación minera implica no solamente establecer una mina, sino varias de ellas en una zona.

11. Todos estos EIAs, deben tener mecanismos de participación en su elaboración.

\section{Distribución y buen uso de la renta minera}

El mayor aporte que puede hacer la minería al desarrollo nacional es el economico-financiero a partir de una distribución justa de la renta generada. Sin embargo, en la actualidad la minera goza de algunas ventajas tributarias para evitar el pago de los impuestos a las sobre ganancias:

- Recuperación adelantada del IGV.

- Contrato de estabilidad tributaria.

- Crédito fiscal por la reinversión.

- $\quad$ El no cobro de regalías.

Por el contrario, se ha establecido el llamado "óbolo minero" o aporte voluntario de apenas $3.75 \%$ de las utilidades extraordinarias para que se paguen los impuestos con dinero, sino con obras escogidas por las empresas. Todo esto ha hecho que el aporte de la minería a los ingresos fiscales sea bastante menor de lo que podría haber sido, en cantidad que pueden sumar 7.941 Millones en el período 2005-2008.

\subsection{Sistema tributario para la minería}

Una mejor distribución de los excedentes mineros, más justo y que permitan que haya un aporte efectivo de la minería al desarrollo nacional, requiere

Oficina Nacional de Procesos Electorales 
atender tanto a las sobre ganancias generadas en los años anteriores, como un sistema tributario adecuado al futuro.

A futuro, un sistema tributario para la minería debe considerar un Sistema General de Regalías aplicables a todos, las que deben regalías ajustarse hacia arriba cuando los precios de los minerales son altos y que también deben ser mayores para aquellas minas de alta ley y bajo costo de extracción.

1. Transparencia financiera que muchas empresas no cumplen.

2. Debe establecerse el Impuesto a las ganancias de capital, inclusive para aquellas transferencias de propiedad. "No puede ser que compra venta de empresas, en la que hay ganancias de cientos de millones de dólares, se realicen sin pagar impuestos en el Perú, pero que sí paguen impuestos en otros países".

3. El Estado debe revisar su forma de participación en el negocio minero.

\subsection{Distribución territorial y canon minero}

Existe una distribución injusta del excedente en la minería, permaneciendo una enorme parte en manos de los capitalistas, respecto a la escasa parte que corresponde al Estado Peruano.

1. También hay una repartición inadecuada entre las distintas regiones, localidades y comunidades. Los recursos naturales pertenecen Constitucionalmente a la Nación.

2. En el corto plazo, resulta conveniente establecer un tope máximo por provincia y distrito, de acuerdo con las necesidades y redistribuir el restante entre quiénes tienen menos y los demás pobres.

3. Rediseñar las fórmulas para repartición de los recursos es también necesario. Una nueva fórmula de repartición debe considerar como beneficiarios aquellos distritos, provincia y regiones pobres, en cuyo espacio aún no hay minas.

\subsection{Salarios, derechos laborales y condiciones de trabajo}

Parte importante de las condiciones para que la minería contribuya al desarrollo humano es que sus trabajadores tengan un trato digno a los Derechos Fundamentales garantizados y una justa distribución de la riqueza, incluye también el pago de salario y condiciones laborales correspondientes al esfuerzo y al riesgo.

1. La libertad sindical, es decir el derecho de los trabajadores a organizarse en sindicatos, reclamar colectivamente y negociar condiciones de trabajo. Impedir el despido de dirigentes por razones de huelga o reclamo.

2. Eliminar los contratos, sub contratos y servicios no personales, que establecen una discriminación y violan los derechos básicos de los trabajadores. Particular atención debe prestarse a los trabajadores que provienen de Comunidades Campesinas. 
3. Las condiciones de la seguridad social por la minería deben ser revisadas, estableciendo la jubilación a edades más tempranas. La salud ocupacional y los accidentes de trabajo deben merecer una mejor atención por parte de empresas y autoridades.

4. Las jornadas atípicas de trabajo, con trabajo continuo de varias semanas sin descanso, deben ser revisadas en trato directo entre empresas y sindicatos.

5. El Estado debe dar alternativas para reducir la probabilidad de despidos, para dar apoyo económico a los despedidos y para facilitar su reubicación.

\subsection{Comunidad campesina y empleo}

Es importante establecer algunas condiciones básicas para que los empleos de comuneros abonen a una relación justa y al desarrollo de las Comunidades Campesinas.

1. El respeto a la autonomía y vigencia de la Comunidad. Las empresas mineras no pueden buscar modificar pactos o amoldar las relaciones con las comunidades a su conveniencia sobre la base de "comprar" comuneros con trabajo.

2. Las negociaciones Comunidad - Mina deben desarrollarse sobre la base de un plan de desarrollo comunal.

3. Los empleos para comuneros y sus condiciones deben establecerse en concordancia con el plan de desarrollo comunal.

4. Las condiciones de trabajo en la minería deben ser dignas y con pleno respeto de los derechos laborales.

\section{Experiencia de resolución de conflictos socio ambientales en el Corredor Minero Sur Andino}

El Proyecto Minero las Bambas, hoy MMG las Bambas, antes del inicio de sus actividades de explotación tenía proyectado la construcción del minero ducto de carácter interregional, de una longitud de 206 kilómetros desde la Planta Concentradora de las Bambas (Cotabambas - Apurímac) hasta la Planta de Molibdeno de Tintaya (Espinar - Cusco), de un ancho de $30 \mathrm{ml}$ como derecho de vía, para el transporte de $193 \mathrm{m3} / \mathrm{h}$ de pulpa de concentrado de cobre y molibdeno para su exportación (5,144 t/d y 32,000 TM/mes), además para transportar un promedio de $25 \mathrm{~L} / \mathrm{seg}$ de agua con el concentrado.

La trayectoria del minero ducto involucraba terrenos para la construcción de zanjas (tubería) y para la instalación de postes de alumbrado eléctrico, más una vía de acceso para la vigilancia y seguridad, nueve estaciones de bombeo, de los cuales cinco estaban ubicadas dentro del territorio de Chumbivilcas, 47 cruces por los ríos y riachuelos, de los cuales 12 cruces por el Río Santo Tomás y 17 cruces por la sub cuenca del río Velille, 19 centros poblados involucrados con un población de 3,563 personas afectadas y 1,148 viviendas, con un área total de 22,490 hectáreas 
afectadas. ${ }^{(3)}$ Pero sin embargo hoy MMG Las Bambas ha descartado la Construcción del minero ducto optando por construir una nueva carretera afirmada en algunas zonas y el uso de las vías públicas, por donde a la fecha transitan decenas y hasta cientos de camiones encapsulados y otros vehículos, dedicados al transporte de concentrado de minerales y otros elementos químicos, de cuyo hecho los pobladores y comunidades campesinas señalan que estarían creando impactos socio ambientales considerables en el trayecto, la que ameritaría se remedie o por lo menos se mitigue dichos daños.

Por su parte la empresa minera HUDBAY PERU S.A.C. con su proyecto minero Constancia, está enclavada entre los distritos de Velille, Livitaca y Chamaca de la Provincia de Chumbivilcas, con sus componentes mineros en su mayor porcentajes en el territorio de Velille, cuya empresa minera tiene como producción con picos de más de 90,000 toneladas por día superando su capacidad proyectada y que al 30 de abril del 2015 , se habían producido 42,575 toneladas de concentrado de cobre, de las cuales aproximadamente 20,500 toneladas fueron embarcadas, lo cual fortalecería su posición como productora de zinc y cobre de alta calidad y de bajo costo $^{(4)}$. De ello se desprende que la Mina y la Planta Concentradora de Constancia ya están operando a la capacidad de diseño o por encima de ésta; pero las comunidades campesinas ubicadas en Áreas de Influencia Directa e Indirecta tiene constantes reclamos de los acuerdos incumplidos a sus compromisos socio ambientales, cuyo reclamo se ha extendido a los distritos y a la propia Provincia de Chumbivilcas.

Sobre el hoy llamado corredor vial Cusco-Apurimac-Arequipa, el gobierno de PPK y su "Gabinete de la Reconciliación", en fecha 11 de Enero de 2018 mediante D.S. $\mathrm{N}^{\circ}$ 006-2018-PCM declaró el Estado de Emergencia por el plazo de 60 días. Luego en fecha 3 de febrero de 2018 es ampliado por 30 días mediante D.S. № 015-2018-PCM, posteriormente en fecha 3 de marzo de 2018 nuevamente es ampliado por otros 30 días mediante D.S. № 025-20128-PCM y recientemente por tercera vez en fecha 12 de abril de 2018 nuevamente es ampliado por 60 días más por el D.S. № 037-2018-PCM.

Con ello se demuestra que el gobierno ve a los Estados de Emergencia como mecanismos adecuados para solucionar los conflictos sociales, siendo dicha medida un acto inconstitucional, al declarar una carretera bajo Estado de excepcionalidad para garantizar y facilitar la inversión minera, en este caso para viabilizar con presencia policial y militar, el transporte y saqueo de los recursos naturales de las tres regiones del país a cargo de una empresa minera transnacional.

Frente a cualquier tipo de problemas de conflictos socio ambientales, las poblaciones afectadas según DE ECHAVE muestran dos tipos de acciones: por una

(3) CooperAcción. Minero ducto del Proyecto Minero Las Bambas. Primera edición, junio 2013.

(4) (Revista MOMENTOS “A toda máquina”, Agosto 2015 N 14/HUDBAY PERU S.A.C., Pág.6) 
parte, conflictos de resistencia o rechazo a la minería como es el caso de Tambogrande, Quilish, Río Blanco, Santa Ana, Conga y Tía María, en los cuales las Mesas de Diálogo no prosperan y las intervenciones por parte del Estado que suelen ser tardías o reactivas al conflicto, en la que por lo general se produce una polarización extrema. Y por otra parte los conflictos de convivencia con la minería, en la que la gente no se opone a la minería, por el contrario la población afectada busca promover vía reclamo al Estado y a las empresas mineras una correcta convivencia entre la minería y población local, es decir, sostener una actividad extractiva que a su vez sea capaz de respetar los derechos económicos, sociales, culturales, ambientales, de salud, de educación, de infraestructura y otras de las poblaciones en el tiempo, casos que se ve en el denominado Corredor Minero del Sur Andino (Cotabambas, Chumbivilcas y Espinar).

De la misma forma, frente a este tipo de problemas socio ambientales se han originado los conflictos mineros y dentro de ello juegan un rol importante las Mesas de Diálogo, Mesas Técnicas o de Desarrollo, precisando que las mesas de dialogo suelen conformarse como respuesta a un conflicto social que ha escalado y ha hecho crisis y busca crear un clima de confianza para llegar a conocer las demandas de fondo y alcanzar acuerdos; mientras las mesas técnicas o de desarrollo, buscan contribuir con el desarrollo local mediante una intervención multisectorial articulada, con la perspectiva de ampliar la presencia del Estado, discutir las soluciones apropiadas y canalizar inversiones y programas públicos, así como los aportes de la Empresa que opera en la zona.

Dentro de este marco existen los mecanismos alternativos de solución de conflictos mineros, como las Mesas de Diálogo, Mesas Técnicas o de Desarrollo, precisando que las mesas de dialogo suelen conformarse como respuesta a un conflicto social que ha escalado y ha hecho crisis y busca crear un clima de confianza para llegar a conocer las demandas de fondo y alcanzar acuerdos; y por su parte las mesas técnicas o de desarrollo, buscan contribuir con el desarrollo local mediante una intervención multisectorial articulada, con la perspectiva de ampliar la presencia del Estado, discutir las soluciones apropiadas y canalizar inversiones y programas públicos, así como los aportes de la Empresa que opera en la zona, de tal manera que este tipo de mesas tendrían lugar previo al surgimiento de un conflicto, pero también es aplicable después del escalamiento del conflicto, precisamente para concertar conjuntamente (sociedad civil - Estado - Empresa) las bases del desarrollo local para el futuro, como es el caso del desarrollo de las Mesas de los distritos de Velille, Chamaca y la propia Provincia de Chumbivilcas, en los cuales por una parte se consolida una Matriz de Proyectos con el Estado y por otra parte la suscripción de Convenios Marco con las Empresas Mineras a través de los aportes sean económicos o no, vía Responsabilidad Social y Ambiental, destinados para diferentes proyectos. Entonces las Mesas de Diálogo serían mayormente de tipo reactivas ya que responden a un conflicto social; Mientras que las Mesas Técnicas o de Desarrollo estarían más ligadas a una estrategia de prevención por lo que se le conoce como preventivas. 


\section{Resultados de la Mesa de Dialogo del Distrito de Velille}

En fecha 27 de Enero del 2016 después del paro preventivo pacífico de 72 horas, se instala la mesa de dialogo del distrito de Velille con la empresa minera Hudbay Peru SAC, incorporando como garante a la Presidencia del Consejo de Ministros, a través de la Oficina Nacional de Diálogo y Sostenibilidad - ONDS, teniendo como resultado haber suscrito, por una parte la matriz de proyectos de inversión para el distrito de Velille con el gobierno central en fecha 27 de octubre del 2016 y por otra parte la suscripción del convenio marco con la Empresa Minera Hudbay Peru SAC en fecha 27 de Diciembre del 2016.

Fruto de los acuerdos de la Mesa de Diálogo, el Gobierno Central a la fecha habría transferido al gobierno local del distrito de Velille más de 38 millones de soles; y por su parte Hudbay Peru SAC ha aportado la suma de S/. 3'500,000.00 por la Responsabilidad Social Empresarial correspondiente al año 2016 con cuyo dinero se han adquirido 07 tractores agrícolas y sus accesorios, así como el financiamiento de la formulación del expediente técnico para el futuro hospital de Velille, la misma que es implementada por el comité de desarrollo distrital de Velille - CDDV, creada dentro del Convenio Marco. Gracias a este Convenio según se tiene la información la Empresa estaría aportando otro monto de dinero. ${ }^{(5)}$

Recientemente, el 2 de mayo de 2018, se ha instalado el "Comité de Dialogo Ampliado para el Desarrollo de Velille, entre la minera Las Bambas y el distrito de Velille", en la que actúan como mediador y facilitador el Ministerio de Energía y Minas y la Secretaría de Gestión Social y Diálogo de la VGT/PCM, proceso de diálogo que está en marcha.

\section{Resultados de la Mesa Técnica para el Desarrollo de la Provincia de Chumbivilcas}

Enfecha 3 de noviembre del 2016, se Instala la Mesa Técnica para el desarrollo para la provincia de Chumbivilcas, bajo la Presidencia Colegiada del Ministro de Energía y Minas y el Alcalde Provincial de Chumbivilcas, habiéndose designado una coordinación colegiada integrada por profesionales chumbivilcanos ${ }^{(6)}$ siendo ejes temáticos: minería y medio ambiente, salud, educación y saneamiento básico, desarrollo económico productivo - transportes y comunicaciones, y ordenamiento y delimitación territorial, los cuales han sido discutidos en varias reuniones, teniendo como resultado haber suscrito por una parte la "matriz del

(5) Acta de Instalación del "Comité de Diálogo ampliado para el desarrollo de Velille, entre La Minera Las Bambas y el distrito de Velille de fecha 2 de mayo de 2018.

(6) Antonio Eliseo Huañahui Sillocca, Milton Barrionuevo Orosco, Ike Eberth Molina Romero y Edwin Contreras Aguirre, 
plan de inversión para la priorización del proyectos para el desarrollo", por la cual el Gobierno Nacional priorizará para Chumbivilcas una inversión de S/. 2,712'656,313 (dos mil setecientos doce millones seiscientos cincuenta y seis mil trescientos trece soles) que se muestra en el siguiente cuadro:

PROYECTOS DE INVERSION POR SECTORES MINISTERIALES

\begin{tabular}{|l|c|l|}
\hline \multicolumn{1}{|c|}{ SECTOR } & PROYECTOS & MONTO INVERSION \\
\hline MINAGRI & 41 & S/. 406' $944,465.43$ \\
\hline MINEDU & 31 & S/. 93'003,703.29 \\
\hline MVCS & 30 & S/. 132'797,063.17 \\
\hline MINSA & 17 & S/. 135'028,773.00 \\
\hline MTC & 16 & S/. 67' $363,329.00$ \\
\hline MINAM & 07 & S/. 2' $518,980.00$ \\
\hline MINEM & 03 & S/. 837' $656,313.89$ \\
\hline TOTAL GENERAL & $\mathbf{1 4 5}$ & \\
\hline
\end{tabular}

RESUMEN PRESUPUESTO (4)

\begin{tabular}{|l|l|}
\hline $\begin{array}{l}\text { PROYECTOS DE INVERSION POR SECTORES (145 } \\
\text { PROYECTOS) }\end{array}$ & S/. 837'656,313.89 \\
\hline CORREDOR MINERO/VIAL APURIMAC - CUSCO & S/. 1,000'000.000.00 \\
\hline CORREDOR ECONOMICO & S/. 875'000.000.00 \\
\hline TOTAL GENERAL & S/. 2,712' $\mathbf{6 5 6 , 3 1 3 . 8 9}$ \\
\hline
\end{tabular}

Por otra parte, el 23 de Abril del 2017, se ha suscrito con el Gobierno Regional los acuerdos finales aprobando la 2 matriz del plan de inversión de proyectos para el desarrollo de la provincia de Chumbivilcas, por la cual el Gobierno Regional priorizará una inversión de $\mathbf{S} / \mathbf{3 6 0} \mathbf{1 4 7 , 9 0 8 . 0 0}$ (trescientos sesenta millones ciento cuarenta y siete mil novecientos ocho soles), la que se muestra en el siguiente Cuadro: 


\begin{tabular}{|l|r|c|l}
\hline \multicolumn{1}{|c|}{ RESUMEN } & \multicolumn{1}{|c|}{$\mathbf{N}^{\circ}$} & \multicolumn{1}{|c|}{ MONTO S/. } & \multicolumn{1}{|c}{ OBS } \\
\hline OBRAS EN CONTINUIDAD & 19 & $32^{\prime} 690,510.00$ & $2017-2018$ \\
\hline $\begin{array}{l}\text { UNIDAD EJECUTORA } \\
\text { SALUD }\end{array}$ & 01 & $9^{\prime} 294,958.00$ & 2017 \\
\hline PROYECTOS SALUD & 09 & $41^{\prime} 096,630.00 \mathrm{G}$ & ESTION MINSA \\
\hline SANEAMIENTO & 04 & $38^{\prime} 229,154.00$ & PROYECTOS NUEVOS \\
\hline EDUCACION & 05 & $25^{\prime} 439,485.00$ & PROYECTOS NUEVOS \\
\hline PROYECTOS & 03 & $131^{\prime} 509,805.00$ & REPRESAS Y \\
EMBLEMATICOS & & & CARRETERAS \\
\hline OBRAS POR IMPUESTO & 09 & $81^{\prime} 887,365.00$ & \\
\hline TOTAL GENERAL & $\mathbf{5 0}$ & $\mathbf{3 6 0 ^ { \prime } 1 4 7 , 9 0 8 . 0 0}$ & \\
\hline
\end{tabular}

\section{Conclusión: Propuesta de nuevo modelo de desarrollo y minería}

Para repensar la relación entre minería y desarrollo, debemos empezar redefiniendo: ¿qué entendemos por desarrollo?, ¿qué tipo de desarrollo queremos? y ¿a qué valores debe este responder?, para ello se requiere un desarrollo:

1. Centrado en la ampliación de las capacidades y libertades de las personas para ampliar sus posibilidades de ser y hacer, de mejorar su calidad de vida.

2. En el cual las inequidades en el acceso a los recursos no condenen a millones a la pobreza.

3. En el cual los derechos humanos sean respetados de tal manera que todos los individuos tengan la seguridad de que estas garantías básicas otorgan para su vida y que el Estado sea la entidad efectivamente garante de esos derechos.

4. Un desarrollo basado en la Comunidad y donde estás, y en especial los Pueblos Indígenas, tengan un espacio amplio de autonomía en la decisión de su propia opción de desarrollo; lo que demanda el respeto y ampliación de los derechos de los Pueblos Indígenas.

5. Un desarrollo basado en el Justicia Social, en el respeto a las diferencias culturales y en el diálogo intercultural. 
6. Un desarrollo en el cual la mejora de la calidad de vida y la ampliación de los derechos humanos vaya de la mano con un equilibrio armonioso con el Medio Ambiente, de tal manera el aire, el agua, los bosques, la biodiversidad, sean respetados y reservados asegurando de esta manera que futuras generaciones puedan gozar también de esos recursos que la naturaleza nos ha brindado.

El desarrollo no es solamente crecimiento económico, sino es educación, salud, calidad de vida, justicia social, relaciones sociales sanas, participación y libertades, identidad cultural y un buen vivir.

Esta opción de desarrollo implica poner en el centro a la gente y no al mercado o a la economía, que puede tener un rol, pero subordinada o instrumental al bienestar de las personas y de los pueblos. Esta opción también implica poner a los sectores populares, las comunidades y pueblos indígenas como los principales sujetos del desarrollo. En esta opción de desarrollo, el Estado tiene que respaldar las opciones de desarrollo alternativo. en este nuevo modelo de desarrollo, las actividades mineras y extractivas, como cualquier otra actividad económica, deben llevarse a cabo solamente sí contribuyen a ese objetivo. La minería y las actividades extractivas no pueden ser el centro de un modelo de desarrollo de este tipo. El Perú debe basar su desarrollo en la diversidad ambiental, geográfica y cultural, que posee y no solamente privilegiando algunas actividades como la minería. La actividad agropecuaria y las comunidades tienen un lugar de primera importancia en un nuevo modelo de desarrollo.

\section{Bibliografía}

DE ECHAVE J., Alejandro Diez, Ludwig Huber, Bruno Revesz, Xavier Ricard y Martín Tanaka. (2009) Minería y conflicto social. Lima: CBC: CIPCA: CIES: IEP.

DIALOGO CON LA JURISPRUDENCIA N 9. Año 2 (2007). Jurisprudencia constitucional sobre Derecho Ambiental. Lima. Primera Edición. Gaceta Jurídica.

FLORES UNZAGA, César (2016). Conviviendo con la minería en el sur andino. Experiencias de las mesas de diálogo y desarrollo de Espinar, Cotabambas y Chamaca. Lima. Primera Edición. CooperaAcción-OXFAM.

GRUPO DE DIALOGO SOBRE LA MINERIA EN COLOMBIA (GDIAM). Propuestas para una Visión Compartida sobre la minería en Colombia. FORDFONDATION - SSRC.

STEFAN, Engel (2014). Alarma de Catástrofe Ambiental. ¿Qué hacer contra la destruvcción deliberada de la unidad entre el ser humano y la naturaleza?. Lima. Primera Edición. 
VILLA, Juan Pablo (2016). Manual de Negociación y Resolución de Conflictos. Define objetivos, resuelve incidencias y obtén resultados. España-Barcelona. Editorial Profit.

ZEISSER POLATSIK. Marco, (2015). La Experiencia de la Mesa de Diálogo en Espinar 2012 - 2013. ¿Un nuevo modelo de gestión de conflicto socio ambiental? Lima. Primera Edición. CooperaAcción-OXFAM- FORD FUNDATION. 